\title{
Quantitative Characterization of Grain Structure and Orientation using Electron Back-Scattered Diffraction Patterns Collected by Serial Sectioning
}

\author{
M. Groeber ${ }^{*}$, Y. Bhandari", M.D. Uchic ${ }^{\ddagger}$, D.M. Dimiduk ${ }^{\ddagger}$, and S. Ghosh ${ }^{\Uparrow}$ \\ *Department of Materials Science and Engineering, The Ohio State University, Columbus, OH \\ 43210, USA \\ $\$$ Air Force Research Laboratory, Materials and Manufacturing Directorate, AFRL/MLLMD, \\ Wright-Patterson AFB, OH 45433-7817, USA \\ ๆ Department of Mechanical Engineering, The Ohio State University, Columbus, OH 43210, USA
}

It is well-known that some parameters such as the true size, true shape, and connectivity of microstructural features cannot be determined from two dimensional (2-D) images [1]. This problem can be overcome if one can obtain a 3D volume representation of the material microstructure. In addition, many stereological calculations yield only average values to describe microstructural features, whereas characterizing the full distribution of these features may be of interest, especially for properties which are affected by the tails of the distribution. The need to more completely characterize microstructure has led to the development of new experimental and computational tools for characterizing microstructure in 3-D.

This paper will present a methodology that allows one to calculate stereological parameters of grain structures from a series of two dimensional (2-D) electron back-scatter diffraction (EBSD) maps. The crystallographic maps were collected using a Dual Beam Focused Ion Beam-Scanning Electron Microscope (FIB-SEM) outfitted with an EBSD system [2,3]. This system was used to serial-section a fine-grained nickel-base superalloy via FIB-milling. An example data set consisted of sectioning a $\sim 40 \times 40 \times 30$ micron volume with an inter-slice thickness of 250 nanometers, and an EBSD map was collected for each section. Custom software scripts were developed to automate the serialsectioning and data collection process.

Data analysis was accomplished using a software program developed in this work called MicroImager. Micro-Imager uses the 2-D EBSD maps to automatically define grains and grain boundaries without user intervention, as shown in Figure 1. Once these microstructural elements are defined, Micro-Mesher outputs stereological parameters such as the grain size, number of neighboring grains, grain orientation, and grain boundary misorientation angle for every 2-D section. At present, MicroImager can be used to examine a series of 2-D EBSD maps from a serial-sectioning experiment, and in this way the characterization of a volume can be performed in a more complete manner. An example of this analysis is shown in Figure 2 for the average and maximum grain area. Although this analysis is not a 'true' 3-D characterization of the serial-sectioning data, this methodology can be used to provide a more quantitative view of the material microstructure. In addition, with information about each microstructural constituent, correlations between parameters can be created. Current efforts are focused on extending this methodology to define and analyze the grain structure in 3-D, and progress in this area will be presented as well. 
[1] R.T. DeHoff, J. Microscopy, Vol. 131 (1983) 259-263.

[2] M. Groeber et.al, Numiform 8 (part 2) (2004) 1712.

[3] M.D. Uchic et.al, Microsc. Mircoanal. 10 (suppl. 2) (2004) 524.

[4] The authors gratefully acknowledge financial support for this work from the Air Force Office of Scientific Research, under the direction of Dr. Craig Hartley.
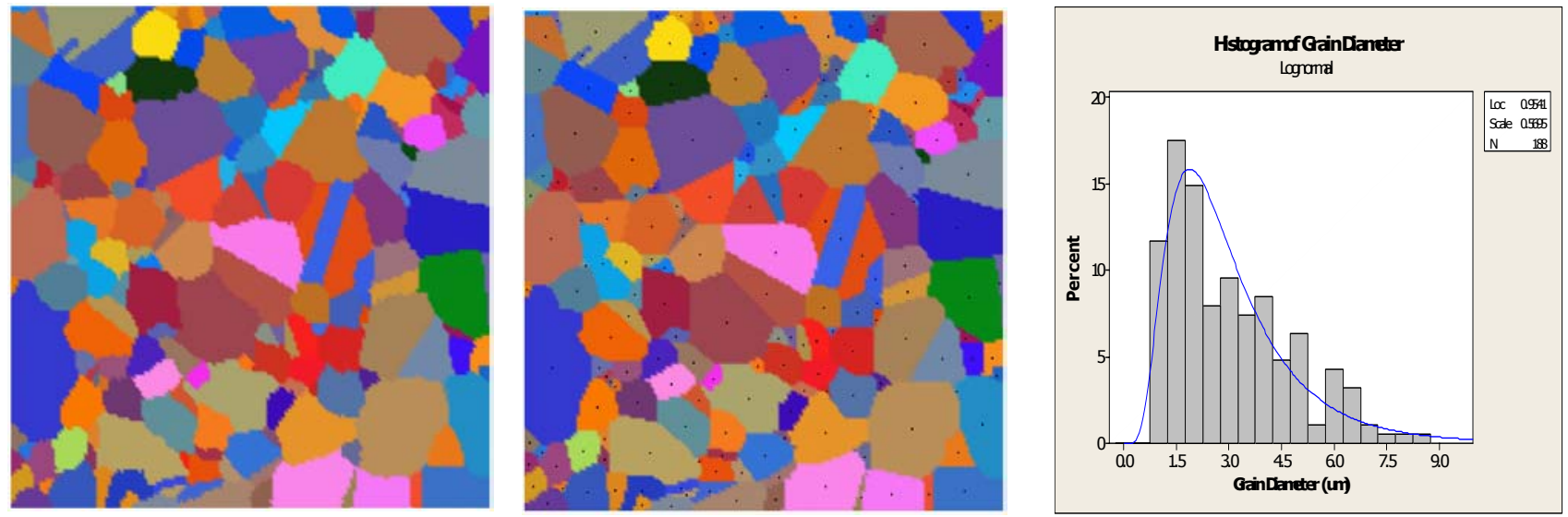

Figure 1. (a) EBSD map from a section of the serial sectioning experiment. Material is a nickelbased superalloy. (b) Grain approximation by Micro-Mesher of the EBSD map in (a). The black dot in the center of each grain is the centroid of that grain. (c) Probability Density Function of grain diameter as calculated from the Micro-Mesher grain map shown in (b).

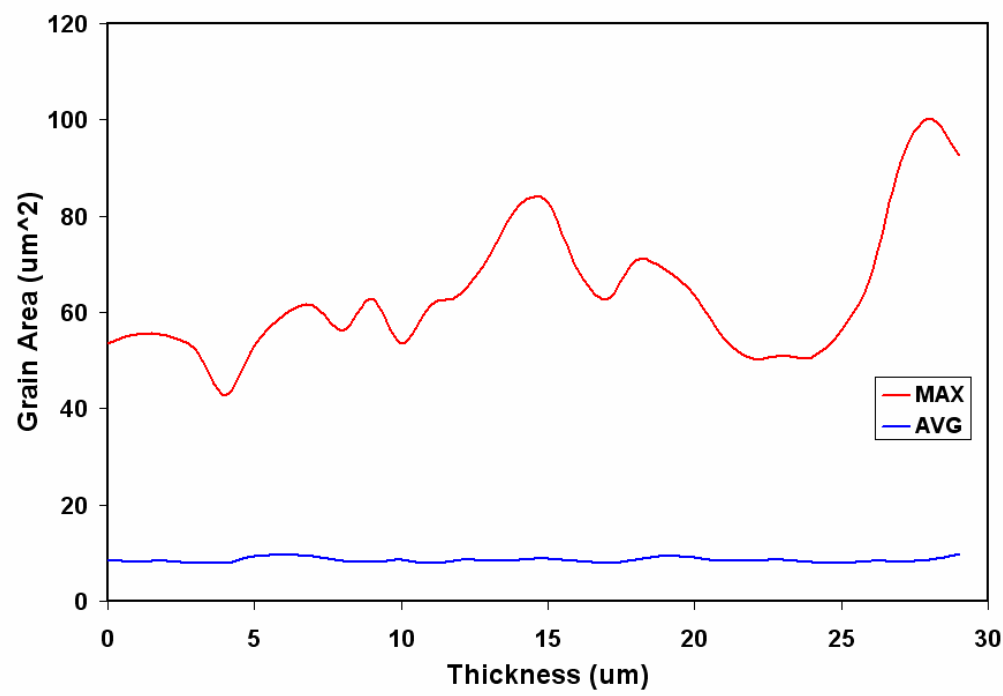

Figure 2. Plot of both the average and maximum grain area for each slice of a serial-sectioning experiment. This figure shows that there is significant variation in the maximum grain area for each section throughout the sample volume, while in comparison the average grain area remains constant. 